\title{
A variant of FGF19 protects the liver from cholestatic injury without inducing cancer
}

New research published in Science Translational Medicine has demonstrated that M70, an engineered variant of fibroblast growth factor (FGF) 19, limits cholestatic liver injury without increasing the risk of developing hepatocellular carcinoma-a risk that is associated with long-term exposure to FGF19.

The authors engineered mice to overexpress M70, FGF19 (a positive control) or green fluorescent protein (a negative control) for 24 weeks. Liver tumours were found in the FGF19 group but not in the M70 or green fluorescent protein groups. In addition, M70 suppressed the expression of $C y p 7 a 1$, a gene that encodes a key enzyme involved in bile acid synthesis. Both results suggest that M70 can regulate bile acids in a similar way to FGF19, without enhancing carcinogenesis.

In two mouse models of cholestasis with severe liver injury, administration of M70 decreased the build-up of intrahepatic bile acids and serum levels of total bile acids compared with placebo. As another indication of injury prevention, M70 reduced the raised serum levels of liver enzymes to that seen at baseline.

The efficacy of M70 in controlling human bile acid synthesis was studied in a randomized, double-blind clinical trial. Healthy individuals were injected with either M70 $(n=9)$ or placebo $(n=11)$ for 7 days. The serum concentration of $7 \alpha$-hydroxy-4-cholesten3-one-a marker of hepatic CYP7A1 activity-was reduced compared with pretreatment measurements.

As well as evaluating M70 as a treatment for diseases related to bile acids, future studies will investigate the effect of M70 on metabolic liver diseases such as NASH.

Gillian Patman

Original article Luo, J. et al. A nontumorigenic variant of FGF19 treats cholestatic liver disease. Sci. Transl. Med. 6, 247ra100 (2014) 